\title{
Process engineering and pharmaceutical manufacturing technologies
}

\author{
Mohammed Maniruzzaman ${ }^{1}$ - Ali Nokhodchi ${ }^{1} \cdot$ Robert O. Williams III ${ }^{2}$ \\ Published online: 5 July 2018 \\ (C) Controlled Release Society 2018
}

In recent years, the pharmaceutical industry is going through a period of exceptional changes, where emerging market expansion and technology advances have increased sector growth. Many new drug candidates found in the drug discovery pipeline generally have poor physicochemical and biopharmaceutical properties, such as poor solubility or low chemical compatibility, which severely limits their oral bioavailability and thus therapeutic performance. The reduction in the numbers of new molecules coming to market along with the expiry of the existing patents is also forcing the pharmaceutical industry to invest in and embrace new technologies that can save both costs and time for manufacture. One timely solution to the growing need can be the exploitation of continuous manufacturing and process engineering with better controls and reproducibility and low labour and production cost. Recently, implementation of process analytical tools (PAT) under the Quality by Design $(\mathrm{QbD})$ paradigm has attracted a strong interest by pharmaceutical scientists as well as the regulatory agencies across the globe. The ultimate aims of these QbD approaches are being the science-based design and development of formulation and manufacturing processes for the predefined product quality objects.

As the guest editors of this special issue of Drug Delivery and Translational Research, we are pleased to introduce our theme issue on "Process Engineering and Pharmaceutical Manufacturing Technologies". This theme issue contains two distinct parts: the first part focuses on recent developments of various novel formulations via continuous manufacturing, i.e. hot melt extrusion (HME), whereas the second part focuses on various aspects of emerging pharmaceutical technologies and their contributions to drug delivery.

Mohammed Maniruzzaman

M.Maniruzzaman@sussex.ac.uk; m.maniruzzaman12@gmail.com

1 Department of Pharmacy (Chemistry), School of Life Sciences, University of Sussex, Falmer, Brighton BN1 9QJ, UK

2 Division of Molecular Pharmaceutics and Drug Delivery, The University of Texas at Austin, PHR 4.214B, 1 University Station, A1920, Austin, TX 78712-0128, USA
Timely and authoritative, this issue contains 15 original research articles and 5 critical review articles that will be of significant interest to readers across both industry and academia.

The paper authored by Wesholowski et al. reported the application of an in-line UV/Vis spectrometer as a PAT tool for early development of Twin-Screw-Extruder and the performance tested in accordance with the International Conference on Harminisation (ICH) Q2 - guideline. In this study, the authors explored two APIs (carbamazepine and theophylline) with one polymer matrix (copovidone) to quantify the drug loading in the formulation. Similarly, Omar et al. studied the temperature of the ribbon via an online thermal camera which was used to explain the differences in ribbon properties at varying process parameters. The authors found that the increase in the roller speed during roller compaction somehow decreased the width of the ribbon while increasing the percentage of fines in the product. In a separate study, Al Asady et al. studied the plastic deformation of various primary particles via roller compaction process by determining two mechanical properties: the nano-indentation hardness and the viscoelasticity of the primary powder. The authors also identified that the plastic deformation of the material had the potential to provide an indication for the ability of the primary powder to produce a good ribbon. Desai et al. implemented a design of experiments (DoE), a tool of QbD paradigm, in order to evaluate the interaction of input variables (compression force, tablet punch configuration and directly compressible (DC) filler) affecting the response factors (tablet hardness and capping rating) during the manufacture of pharmaceutical tablets. Similarly, Pawar et al. investigated the dissolution rate performance of amorphous solid solutions of a poorly watersoluble drug, efavirenz (EFV), in amorphous polymeric matrices by adopting a Box-Behnken design of experiment (DoE) approach. Box-Behnken factorial design was conducted to evaluate the effect of independent variables such as polymer ratio, HME screw speed and processing temperature on responses such as solubility and dissolution rate.

In another study, Majumder et al. explored the first case of the implementation of solution calorimetry (SolCal) in order 
to determine the amorphous content of crystalline drug in the amorphous granules prepared via emerging single-step continuous twin-screw dry granulations (TSG). The authors claimed to have made an attempt to interrelate the findings from the SolCal to that of the release of the drug. Li et al. presented a new polymer blend system of ethylcellulose (EC) with poly(vinylpyrrolidone-co-vinyl acetate) (PVP VA64) for sustained release prepared via HME whereas Sawant et al. explored the use of Eudragit ${ } S 100$ and Eudragit ${ }^{\circ} L 100$ alone or in combination with release-modifying agent, namely PolyoxTM WSR 303 and EudragitßL100-55 for delayed release dosage forms via HME processing. A critical review in the area of sustained-release dosage forms authored by Feng et al. provides a comprehensive report on the formulation composition and processing conditions of twin-screw extrusion and how these factors affect the drug release properties of sustained-release dosage forms whereas Maincent et al. reviewed the details of the challenges and the formulation approaches employed during the manufacture of sustainedrelease amorphous solid dispersions (ASDs). Another review paper authored by Chavan et al. describes various continuous manufacturing methods for co-crystal production, along with in-line monitoring during co-crystal production, emphasising on process analytical technology (PAT) as well as the scale up issues of continuous and batch co-crystallisation and other suitable techniques for pharmaceutical scale up. Brunaugh et al. critically discuss the optimization of the pharmaceutical milling process through design-space development, theoretical and empirical modelling, and monitoring of critical quality attributes.

Ahmed et al. developed a calcium alginate (CA) wafer dressings by lyophilisation of hydrogels to deliver ciprofloxacin (CIP) directly to the wound site of infected diabetic foot ulcers (DFUs) whereas Molina et al. utilised optimised spray drying process to modify the physicochemical/mechanical properties of lactose-based particles to enhance the aerosolisation performance of salbutamol sulphate in the presence of leucine in the spray dried powders. Trivedi et al. reported a study that considers the in vitro uptake and release characteristics of the binary theophylline-smectite system with a maximum uptake of $67 \pm 2 \mathrm{mg} \mathrm{g}^{-1}$. In a separate study, the group developed polycaprolactone (PCL) nanoparticles via supercritical fluid extraction of emulsions (SFEE) using supercritical carbon dioxide $\left(\mathrm{scCO}_{2}\right)$. Jara et al. studied the process to yield monomodal and uniform polymeric nanoparticles via a nanoprecipitation method. To further identify key variables and their interactions, the authors used artificial neural networks (ANN) to investigate the multiple variables which influence the process. In another study, Fritz et al. developed a novel lipid-core micelle (LCM) as a nanocarrier to encapsulate a poorly water-soluble drug, nifedipine (NFD), by hot melt emulsification, a low-energy method. Haj-Ahmad et al. designed, engineered and utilised a novel multi-tip emitter (MTE) device for potential up scaled electrohydrodynamic atomisation (EHDA), an emerging technique for the production of micron and nanoscaled particles of ketoprofen-a model drug - by comparison with a conventional singleneedle system. An exhaustive review paper authored by Tarbox et al. highlights recent developments in microneedle coating and manufacturing techniques, including micro-milling, atomized spraying, inkjet printing, drawing lithography, droplet-born air blowing, electro-drawing, continuous liquid interface production, 3D printing and polyelectrolyte multilayers coating.

The guest editors of this theme issue would also like to thank the authors for their valuable contributions and input without which this theme issue would not have been possible. It is through their persistent and collective efforts that such a comprehensive and insightful theme issue was created. 